\title{
Protective effect of interleukin-10 and recombinant human keratinocyte growth factor-2 on ventilation-induced lung injury in rats
}

\author{
J.Y. Wu, Z.H. Xiong, G.Z. Xiong, F.Q. Ding, J. Lei, S. Lu, Y. Li, \\ G.M. He, L.L. Zhao and Z.J. Liu \\ Department of Intensive Care Unit, Sichuan Cancer Hospital, Chengdu, China \\ Corresponding author: Z.J. Liu \\ E-mail: zhenjunsichuan@163.com
}

Genet. Mol. Res. 14 (4): 15642-15651 (2015)

Received July 6, 2015

Accepted September 28, 2015

Published December 1, 2015

DOI http://dx.doi.org/10.4238/2015.December.1.16

\begin{abstract}
A rat model of ventilation-induced lung injury (VILI) during anesthesia was generated to investigate the potential role and possible mechanism of interleukin-10 (IL-10) and recombinant human keratinocyte growth factor-2 (rhKGF-2) in protecting anesthetized rats against VILI. A total of 50 male SD rats were randomly divided into 5 groups $(\mathrm{N}=10$ each): control, VILI, IL-10, rhKGF-2, and IL-10 + rhKGF-2. The VILI (model) group was generated via ventilation, with a tidal volume of $20 \mathrm{~mL} / \mathrm{kg}$. Rats in the IL-10 and rhKGF-2 groups received $8 \mathrm{mg} / \mathrm{kg} \mathrm{IL}-10$ and $5 \mathrm{mg} / \mathrm{kg} \mathrm{rhKGF}-2$, respectively, prior to ventilation. The rats in the IL-10 + rhKGF-2 group received both $8 \mathrm{mg} /$ $\mathrm{kg}$ IL-10 and $5 \mathrm{mg} / \mathrm{kg}$ rhKGF-2 $72 \mathrm{~h}$ before ventilation. The total number of nucleated cells and neutrophils in the bronchial alveolar lavage fluid was quantified, and the pathological changes in the pulmonary tissues examined by hematoxylin and eosin staining. The transcript and protein levels of surfactant protein C (SP-C) in lung tissues were detected by real-time polymerase chain reaction and western blot analyses. The
\end{abstract}


$S P-C$ mRNA expression in both IL-10 and rhKGF-2 groups was similar to that in the VILI group. However, this was significantly elevated in the combined treatment group $(\mathrm{P}<0.05)$, indicating that IL-10 and rhKGF-2 could synergistically protect the lung tissue from VILI via the enhancement of $S P-C$ mRNA expression in lung tissues. The protein assay showed a decreased level of infiltration and activation of inflammatory cells, in addition to increased expression of SP-C, thereby confirming the efficacy of this treatment in preventing VILI during anesthesia.

Key words: IL-10; rhKGF-2; Ventilation; Lung injury; Lung protection

\section{INTRODUCTION}

Mechanical ventilation (MV), a basic life support method used during anesthesia, plays a key role in the treatment of critical patients, especially those with acute lung injuries and acute respiratory distress syndrome (Ghanbarpour et al., 2014; Tsushima and Tatsumi, 2014). MV has both positive and negative effects in the field of anesthesia medicine; MV improves the functions of critical organs, including the heart, brain, liver, and kidney, thereby stabilizing the inner environment of the body and facilitating patient recovery. Conversely, MV results in ventilation-induced lung injury (VILI) during anesthesia, causing injuries to various pulmonary tissues, including volutrauma, barotrauma, and lung atelectasis. MV could even worsen the disease, leading to death due to multiple organ failure, thereby severely affecting the prognosis (Alam et al., 2014; Chacon-Cabrera et al., 2014; Retamal et al., 2014). The incidence rate of VILI in anesthesia of patients under MV is as high as $15 \%$, resulting in a higher percentage of death than that caused by hypoxia (Carvalho et al., 2014; Futier et al., 2014). Therefore, it is critical (in anesthesia medicine) to elucidate the pathogenesis of VILI, and to determine effective preventive measures.

VILI can be induced by MV or pulmonary disease in a sequential process including the overexpansion of pulmonary tissues, periodic re-expansion of alveolar cells, recruitment of inflammatory cells after lung injury, increased production of inflammatory factors, damage to the alveolar epithelial cell membrane, and elevated cell apoptosis, which further facilitates the occurrence and development of VILI (Moraes et al., 2014; Saddy et al., 2014). Alveolar surfactants are responsible for a range of biological responses, which could prevent the activation of inflammatory cells and inhibit the release of inflammation-related cytokines, thereby modulating the immune response. The quantity and quality of alveolar surfactants are known to change significantly during $\mathrm{MV}$; this results in the inactivation of inflammatory biological responses, thereby facilitating the advancement of VILI (Kneyber et al., 2014; Park et al., 2014). Therefore, current research is focusing on identifying the factors that could regulate alveolar surfactants and improve VILI. Previous studies have revealed that interlukin-10 (IL-10) and recombinant human keratinocyte growth factor-2 (rhKGF-2) could facilitate mitosis in type II alveolar epithelial cells and ameliorate pulmonary tissue damage (Cross et al., 2013; Hokenson et al., 2013; Yang et al., 2014). The synergistic effect of IL-10 and rhKGF-2 in anesthetized patients, and the possible mechanism of action, however, remain to be elucidated. Therefore, this study investigated the functional role and possible mechanism of IL-10 and rhKGF-10 in anesthetized MV model rats. 


\section{MATERIAL AND METHODS}

\section{Experimental animals}

Two-month-old healthy male SD rats (specific pathogen free (SPF)-grade, body weight $250 \pm 20 \mathrm{~g}$ ) were purchased from the animal center of Sichuan University, and were kept in an SPF-grade animal experiment center. The study protocols were pre-approved by the Animal Experiment Ethics Committee of Sichuan University.

\section{Reagents}

rhIL-10 and rhKGF-2 were purchased from Life Technologies (Carlsbad, CA, USA) and diluted to concentrations of 8 and $5 \mathrm{mg} / \mathrm{kg}$, respectively, with sterilized phosphate-buffered saline (PBS); the diluted protein solutions were stored at $-20^{\circ} \mathrm{C}$ until further use. Chloral hydrate $(10 \%)$ was provided by the Central Laboratory of our hospital. The reagents for hematoxylin and eosin (HE) staining and western blot analysis were obtained from Beyotime Institute of Biotechnology (Shanghai, China). The polyvinylidene fluoride (PVDF) membrane was purchased from Pall Life Sciences (Port Washington, NY, USA). Ethylenediaminetetraacetic acid (EDTA) was purchased from HyClone Laboratories, Inc. (South Logan, UT, USA). ECL reagents were purchased from Amersham Biosciences (Amersham, UK). TRIzol reagent, the RNA extraction kit, RT-PCR primers, reverse transcription kit, and real time-PCR reagents were obtained from Invitrogen (Carlsbad, CA, USA). Rabbit anti-human surfactant protein $\mathrm{C}$ (SP-C) antibody and horseradish peroxidase (HRP)-conjugated mouse anti-rat IgG were purchased from Cell Signaling Technology (Danvers, MA, USA). The $\beta$-actin antibody was obtained from Santa Cruz Biotechnology (Dallas, TX, USA). The animal ventilator was obtained from Harvard University (USA), while the tracheal intubation tube and carotid artery cannula were purchased from BD Biosciences (Franklin Lakes, NJ, USA).

\section{Animal models}

Fifty male SD rats were divided into 5 groups $(\mathrm{N}=10$ per group): control group, VILI group, IL-10 group, rhKGF-2 group, and the IL-10 + rhKGF-2 group. The anesthetized rats of the VILI group were subjected to MV via tracheal intubation, using the following parameters: $21 \% \mathrm{O}_{2}, 0 \mathrm{~cm} \mathrm{H} \mathrm{H}_{2} \mathrm{O}$ of positive end-expiratory pressure, $20 \mathrm{~mL} / \mathrm{kg}$ tidal volume, and RR maintained at 70/min. The animals were sacrificed after $4 \mathrm{~h}$. The animals in the IL-10 and rhKGF-2 groups were provided with $8 \mathrm{mg} / \mathrm{kg}$ rhIL-10 and $5 \mathrm{mg} / \mathrm{kg}$ rhKGF-2; the animals were then prepared as described for the VILI group. The rats in the IL-10 + rhKGF-2 group were injected with $5 \mathrm{mg} / \mathrm{kg}$ rhKGF-2 and $8 \mathrm{mg} / \mathrm{kg}$ rhIL-10 at $72 \mathrm{~h}$ prior to the induction of VILI. Animals in the control group received tracheal intubation (without MV) after anesthesia.

\section{Differential count of bronchial alveolar lavage fluid (BALF) cells}

Two milliliters of $1 \mathrm{X}$ PBS was injected into rat left pulmonary tissues via the tracheal intubation tubes. After three repeated elutions, the lavage fluid was collected and centrifuged 
at $4^{\circ} \mathrm{C}$ at $200 \mathrm{~g}$ for $10 \mathrm{~min}$ in order to obtain a fluid cell suspension. These mixtures were then applied to cell-counting slides, which were centrifuged and differentially counted under the microscope.

\section{HE staining of pulmonary tissues}

The animals were sacrificed and dissected in order to collect the lower lobular tissues of the right lungs. Tissue samples were fixed in $4 \%$ neutral-buffered formaldehyde for $48 \mathrm{~h}$, dehydrated in gradient ethanol, subjected to hyalinization, and immersed in paraffin. Samples were then embedded in wax and sectioned. The samples were dewaxed using routine procedures, and the tissue slides stained with HE; the slides were then observed under the microscope. Residual pulmonary tissues were frozen at $-80^{\circ} \mathrm{C}$ for further use.

\section{Detection of $S P-C$ mRNA levels using real-time PCR}

Frozen pulmonary tissue samples were grinded on ice, using a sterilized tissue grinding rod. The tissues were repeatedly eluted with $150 \mu \mathrm{L}$ buffer after complete homogenization until a clear solution was obtained. Tissue suspension fluid was then transferred to sterilized centrifuge tubes, which were then centrifuged at 20,000 rpm for $2 \mathrm{~min}$. The supernatant was transferred to newly sterilized tubes. The lung tissue mRNA was extracted using TRIzol reagent, according to the manufacturer instructions. cDNA was synthesized using mRNA as the template and specific $S P-C$ primers. Real-time PCR was performed to quantify the transcript level of the target gene. The following primer sequences were used for real-time PCR: SP-C-F, 5'-CTCGT AGCGA CAGAT CGCTAG-3'; SP-C-R, 5'-TAGGC CTGCC GTCCC GTCA-3'; GAPDH-F, 5'-GACTG GAAGC CTGTT AGCT-3'; GAPDH-R, 5'-CGTTA GACTG GAGAT GTCGG A-3'. The reaction conditions were set as follows: $55^{\circ} \mathrm{C}$ for $1 \mathrm{~min} ; 36$ cycles of denaturation at $95^{\circ} \mathrm{C}$ for $30 \mathrm{~s}$, annealing at $56^{\circ} \mathrm{C}$ for $45 \mathrm{~s}$, and elongation at $72^{\circ} \mathrm{C}$ for $35 \mathrm{~s}$. The $\mathrm{Ct}$ values were determined using GAPDH as the internal reference, with an Opticon-2 fluorescence quantitative PCR reactor; the level of mRNA expression was determined by the $2^{-\Delta \Delta C t}$ method, from the standard curve.

\section{Determination of the SP-C protein content in lung tissues}

Pulmonary tissue proteins were extracted from the lysis solutions, which were subjected to centrifugation at $10,000 \mathrm{rpm}$ and $4^{\circ} \mathrm{C}$ for $15 \mathrm{~min}$. The proteins were separated by $10 \%$ sodium dodecyl sulfate polyacrylamide gel electrophoresis; the separated proteins were transferred to PVDF membranes and blocked with 5\% skim milk at room temperature. The membranes were incubated with monoclonal anti-SP-C antibody (1:500), followed by incubation with 1:2000 HRP-conjugated mouse anti-rabbit IgG. The membranes were incubated in the dark for $30 \mathrm{~min}$ at room temperature, and subsequently developed for $1 \mathrm{~min}$ using electrochemiluminescence reagents. The membranes were then exposed to X-rays. The Bio-Rad image processing system (Hercules, CA, USA) was used to analyze the results obtained, and the relative protein density calculated using the Quantity One software (Bio$\mathrm{Rad})$. Anti-actin antibody was employed as an internal reference to validate sample volume in each group. All experiments were repeated four times. 


\section{Statistical analysis}

The SPSS v.12.0 software package (IBM, Armonk, NY, USA) was used to analyze all data obtained; the enumeration data were processed by the chi-squared test, while the measurement data are reported as means \pm standard deviation (SD). One-way analysis of variance (ANOVA) was employed for analysis of multiple groups. $\mathrm{P}$ values $<0.05$ were considered to be statistically significant.

\section{RESULTS}

\section{Cell count in rat BALF and the effects of IL-10 and rhKGF-2}

BALF was obtained from rats of all groups; the total number of nucleated cells and neutrophils were counted. The results revealed significantly elevated levels of total nucleated cells and neutrophils in rat BALF from the VILI group, compared to those from the control group $(\mathrm{P}<0.05)$. Single rhKGF-2 or rhIL-10 administration reduced the total number of inflammatory cells to a certain extent; however, this reduction was without statistical significance, compared to the VILI group. The combined application of rhKGF-2 and rhIL10 , however, resulted in a statistically significant reduction in the number of total nucleated cells and neutrophils in rat BALF compared to the animals of the VILI group $(\mathrm{P}<0.05)$. The level of inflammatory cells in the combined treatment group was significantly higher than that in control rats $(\mathrm{P}<0.05)$, although showing a small level of recovery from VILI. A detailed summary of the cell counts in all groups is provided in Table 1. These results suggest that VILI resulted in elevated inflammatory cell expression, which could be reduced significantly by the combined application of rhKGF-2 and rhIL-10. These results collectively suggested the protective role of IL-10 and rhKGF-2 against VILI.

$\begin{aligned} & \text { Table 1. Total nucleated cell and neutrophil counts in rat bronchial alveolar lavage fluid [means } \pm \text { standard } \\
& \text { deviation (SD)]. }\end{aligned}$
\begin{tabular}{lccc}
\hline Group & $\mathrm{N}$ & No. of nucleated cells $\left(\mathrm{x} 10^{5} / \mathrm{mL}\right)$ & Neutrophil count $\left(\mathrm{x} 10^{5} / \mathrm{mL}\right)$ \\
\hline Control & 10 & $2.5 \pm 0.32$ & $0.71 \pm 0.09$ \\
VILI & 10 & $8.9 \pm 0.92^{*}$ & $4.8 \pm 0.41^{*}$ \\
IL-10 & 10 & $7.1 \pm 0.35^{*}$ & $3.9 \pm 0.37^{*}$ \\
rhKGF-2 & 10 & $6.5 \pm 0.57^{*}$ & $3.2 \pm 0.34^{*}$ \\
IL-10 + rhKGF-2 & 10 & $4.7 \pm 0.65^{* *}$ & $1.98 \pm 0.72^{* *}$ \\
\hline
\end{tabular}

$* \mathrm{P}<0.05$ compared to the control group. ${ }^{\#} \mathrm{P}<0.05$ compared to the VILI group.

\section{Pathological changes in pulmonary tissues}

The pathological changes in the pulmonary tissues of rats of all groups were detected by HE staining. The results showed intact lung structure without inflammatory cell infiltration or alveolar edema in controlled rats. VILI rats, however, featured typical VILI phenotypes, including thickened alveolar septum and edema, and inflammatory cell and neutrophil infiltration into the pulmonary tissues. The IL-10 group displayed significant lung injuries with abundant infiltration of inflammatory cells. Treatment with rhKGF-2 decreased the 
pulmonary damage to some extent, as seen by the reduced inflammatory cell infiltration. The combined usage of IL-10 and rhKGF-2, however, significantly reduced the signs of rat lung tissue damage, as evidenced by decreased pulmonary edema, thinning of the alveolar septum, lower infiltration of inflammatory cells, and decreased exudation of neutrophils (Figure 1A-E).

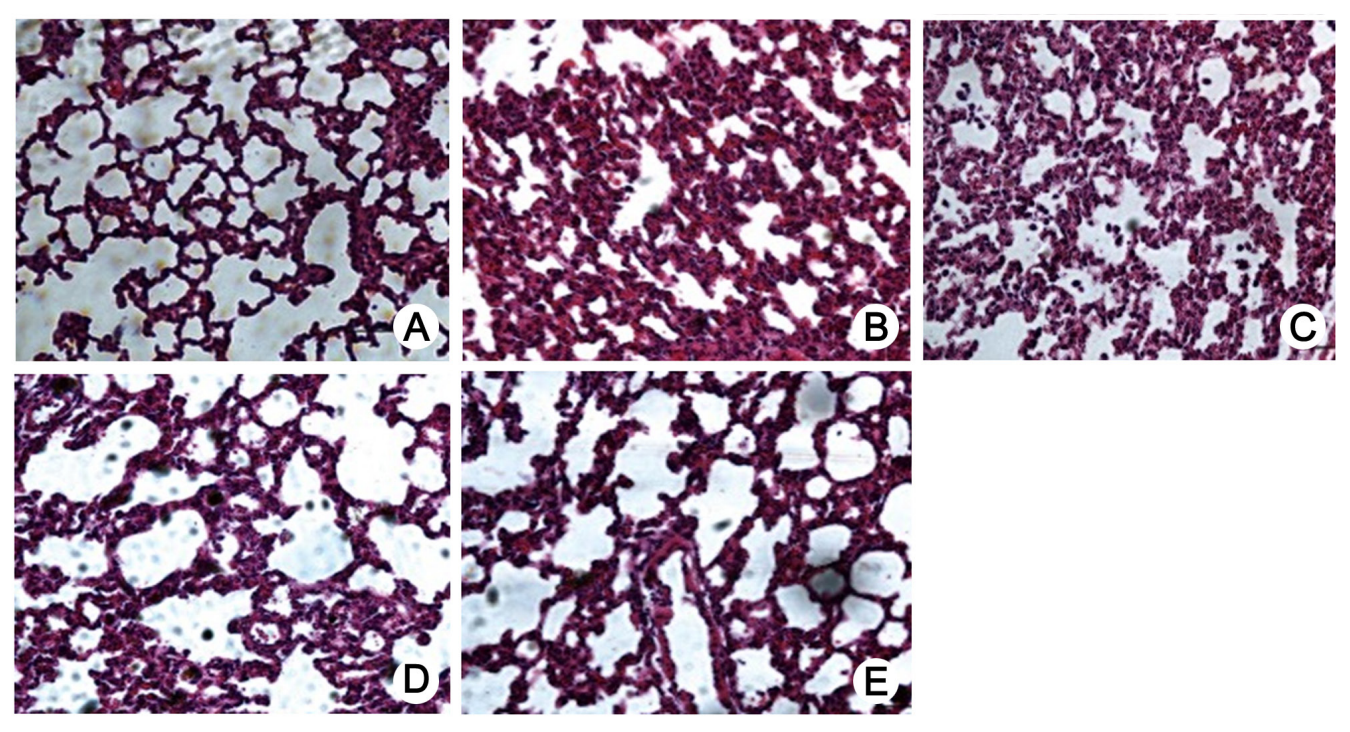

Figure 1. Pathological changes in pulmonary tissues (200X). A. Control group; B. VILI group; C. IL-10 group; D. rhKGF-2 group; E. IL-10 + rhKGF group.

\section{$S P-C$ mRNA expression in rat pulmonary tissues}

The expression profiles of $S P-C$ mRNA in lung tissues of rats from all groups were measured by RT-PCR. As seen in Figure 2, the VILI rats showed a statistically significant decrease in $S P-C$ transcript expression compared to control rats $(\mathrm{P}<0.05)$. The use of rhKGF-2 or rhIL-10 (individually) recovered $S P-C$ mRNA expression to some extent compared to the VILI group, but without statistical significance. The combined application of rhIL-10 and rhKGF-2, however, (statistically) significantly elevated the $S P-C$ mRNA levels in rat pulmonary tissues compared to the VILI rats $(\mathrm{P}<0.05)$. These results collectively suggested that MV causes pulmonary tissue damage via the decreased expression of $S P-C$ mRNA, which can be restored by the combined application of IL-10 and rhKGF-2, which protect the lung tissues.

\section{SP-C protein expression in rat lung tissues}

We further investigated SP-C protein expression in the pulmonary tissues of rats of all groups. The results were consistent with those from real-time PCR (Figures 3 and 4). The SP-C protein level was significantly decreased in the VILI group $(\mathrm{P}<0.05)$. The combined treatment with IL-10 and rhKGF-2 significantly elevated SP-C protein levels $(\mathrm{P}<0.05)$, while 
the individual application of either caused a minor increase in SP-C protein levels, which was not statistically significant. In summary, the consistent SP-C mRNA and protein levels in the different type of rats suggested that IL-10 and rhKGF-2 synergistically protected the lung tissues by facilitating the expression of SP-C.

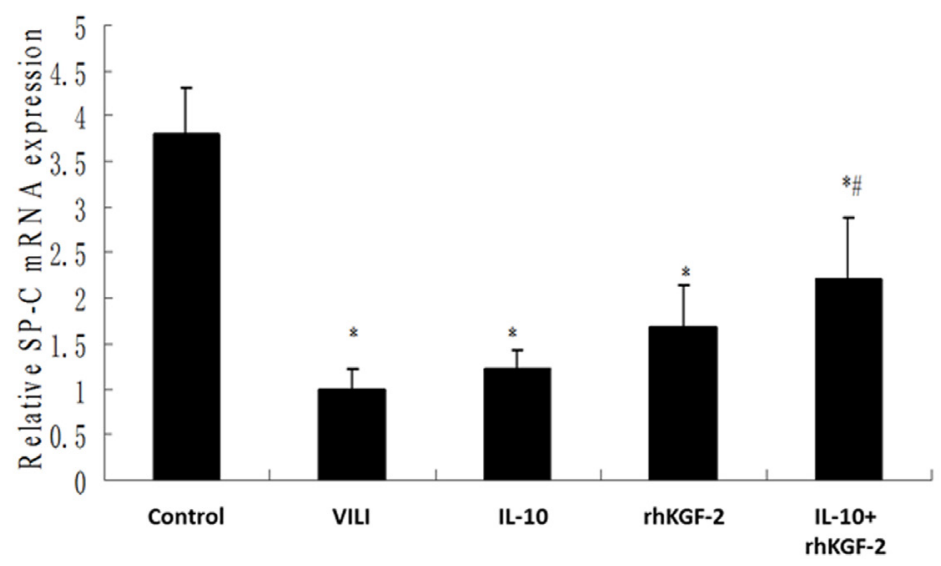

Figure 2. Effects of IL-10 and rhKGF-2 on $S P-C$ mRNA levels. ${ }^{*} \mathrm{P}<0.05$ compared to the control group. ${ }^{*} \mathrm{P}<0.05$ compared to the VILI group.

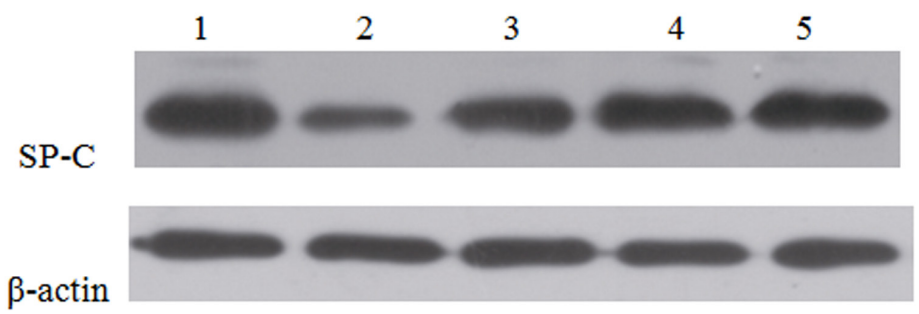

Figure 3. Effects of IL-10 and rhKGF-2 on SP-C protein levels in rat pulmonary tissues. Lane 1 = control group; lane 2 =VILI group; lane $3=\mathrm{IL}-10$ group; lane 4 = rhKGF-2 group; lane $5=\mathrm{IL}-10+$ rhKGF-2 group.

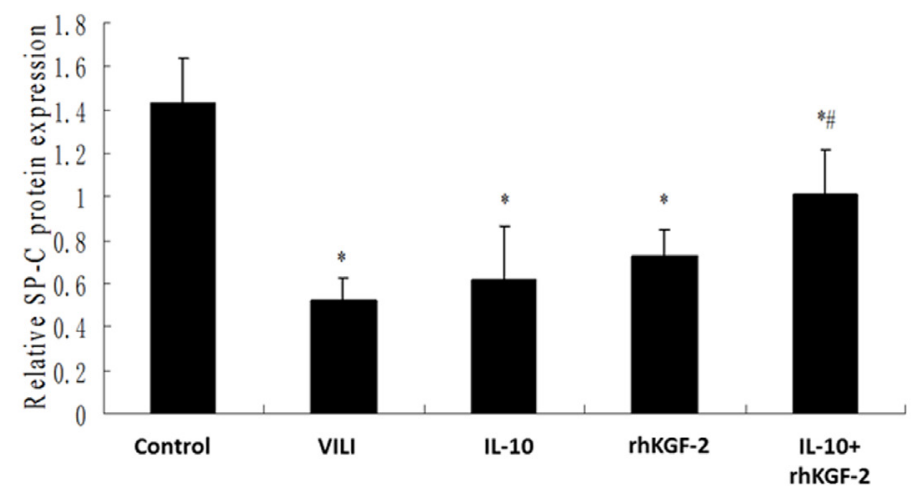

Figure 4. Relative expression levels of the SP-C protein in all groups. ${ }^{*} \mathrm{P}<0.05$ compared to the rats in the control group. ${ }^{\#} \mathrm{P}<0.05$ compared to the VILI group. 


\section{DISCUSSION}

MV-induced pulmonary damage during anesthesia frequently leads to impairment in the critical organs, or even multiple-organ dysfunction syndrome; therefore, this has been a major focus of research over the past few years. The pathogenesis of VILI includes a sequence of biological reactions, ranging from the over-expansion of lungs, mechanical stimulation of pulmonary cells, signal transduction, secretion of chemoattractants, recruitment of inflammatory cells (such as neutrophils), release of cytokines and inflammatory factors by immune cells, occurrence of inflammation, and, finally, pulmonary tissue damage. Nucleated cells, such as polymorphonuclear cells and macrophages participate in early inflammatory response during the initiation and advancement of VILI. Further mechanical stimulation induces the recruitment of abundant neutrophils to the alveolar tissues, and the release of inflammatory factors. One prominent example is the tumor necrosis factor (TNF), which can induce the expression of cell selectin by microvascular endothelial cells to interact with the selectin of neutrophils, whose activation further leads to cell adhesion, migration, and translocation across vascular vessels, and subsequently, recruitment to the pulmonary inflammatory sites, thereby further advancing the injuries to the lung (Amado-Rodriguez et al., 2013; Muller-Redetzky et al., 2014; Shu et al., 2014). The results of this study confirmed that BALF from the VILI rats had significantly elevated numbers of nucleated cells and neutrophils, which were significantly decreased after the combined application of rhKGF-2 and rhIL-10, suggesting the presence of higher levels of inflammatory cells during VILI.

IL-10, an activating factor for B lymphocytes, exerts a negative regulatory role in innate immune response, as it can inhibit secretion of inflammatory factors, such as IL-1 and TNF- $\alpha$, thereby limiting the inflammatory response and preventing lung injuries (Hoegl et al., 2009). KGF-2 specifically facilitates the proliferation, differentiation, and migration of epithelial cells, in addition to enhancing the embryonic development via induction of various signal pathways. In clinical practice, rhKGF-2 can be applied as a cytokine for the treatment of surgical wounds, diabetic ulcers, pancreatitis, and tissue wounds. In addition, rhKGF-2 is known to protect against pulmonary injuries, via the stimulation of mitosis in type II alveolar epithelial cells (Bi et al., 2014; Santos et al., 2014). The potential role and possible synergistic mechanism of IL-10 and rhKGF-2 in the treatment of VILI, however, remains to be elucidated. This study revealed that the combination of rhKFG-2 and rhIL-10 significantly reduced the numbers of inflammatory cells, thereby playing a protective role in VILI.

Pulmonary surfactants (PS) are mixtures composed of lipids and carbohydrates that are synthesized and secreted by type II alveolar epithelial cells. PS helps maintain normal pulmonary structures and functions via its pluripotent roles, such as decreasing alveolar surface tension, drying of alveolar tissues, preventing pulmonary edema, relaxing smooth muscles, participating in local immunity (defense), and improving the properties of slime. As the only cell that produces SP-C, type II alveolar epithelial cell can be specifically marked with SP-C (Roldan et al., 2014; Simonato et al., 2014). This study confirmed a significantly lowered expression level of SP-C mRNA and protein in VILI rats; the SP-C levels in these rats were restored by the combined administration of IL-10 and rhKGF-2. This suggested that MV may cause pulmonary damages because of the decreased expression of SP-C. These results, in turn, indicate the protective role of IL-10 and rhKGF-2, when administered in combination to the pulmonary tissues. 
In summary, MV has been shown to decrease SP-C expression in pulmonary tissues during anesthesia, which results in lung injuries. On the contrary, IL-10 and rhKGF-2 exert a protective function against VILI via the enhancement of SP-C expression in lung tissues. Therefore, this study illustrates the protective role and elucidates the possible mechanism of IL-10 and rhKGF-2 action against VILI, and provides a novel method for the treatment of VILI during anesthesia in clinical practice.

\section{Conflicts of interest}

The authors declare no conflict of interest.

\section{ACKNOWLEDGMENTS}

We thank the anonymous reviewers for reviewing this manuscript.

\section{REFERENCES}

Alam M, Jones G, Kahl W and Kamath MV (2014). Modeling the weaning of intensive care unit patients from mechanical ventilation: a review. Crit. Rev. Biomed. Eng. 42: 25-61.

Amado-Rodriguez L, Gonzalez-Lopez A, Lopez-Alonso I, Aquirre A, et al. (2013). Anti-inflammatory effects of clarithromycin in ventilator-induced lung injury. Respir. Res. 14: 52.

Bi J, Tong L, Zhu X, Yang D, et al. (2014). Keratinocyte growth factor-2 intratracheal instillation significantly attenuates ventilator-induced lung injury in rats. $J$. Cell Mol. Med. 18: 1226-1235.

Carvalho NC, Guldner A, Beda A, Rentzsch I, et al. (2014). Higher levels of spontaneous breathing reduce lung injury in experimental moderate acute respiratory distress syndrome. Crit. Care Med. 42: e702-715.

Chacon-Cabrera A, Rojas Y, Martinez-Caro L, Vila-Ubach M, et al. (2014). Influence of mechanical ventilation and sepsis on redox balance in diaphragm, myocardium, limb muscles, and lungs. Transl. Res. 164: 477-495.

Cross LJ, O'Kane CM, McDowell C, Elborn JJ, et al. (2013). Keratinocyte growth factor in acute lung injury to reduce pulmonary dysfunction - a randomised placebo-controlled trial (KARE): study protocol. Trials 14: 51.

Futier E, Godet T, Millot A, Constantin JM, et al. (2014). Mechanical ventilation in abdominal surgery. Ann. Fr. Anesth. Reanim. 33: 472-475.

Ghanbarpour R, Saghafinia M, Ramezani Binabaj M, Madani SJ, et al. (2014). Pulmonary infections in ICU patients without underlying disease on ventilators. Trauma Mon. 19: e15958.

Hoegl S, Boost KA, Czerwonka H, Dolfen A, et al. (2009). Inhaled IL-10 reduces biotrauma and mortality in a model of ventilator-induced lung injury. Respir. Med. 103: 463-470.

Hokenson MA, Wang Y, Hawwa RL, Huang Z, et al. (2013). Reduced IL-10 production in fetal type II epithelial cells exposed to mechanical stretch is mediated via activation of IL-6-SOCS3 signaling pathway. PLoS One 8: e59598.

Kneyber MC, Zhang H and Slutsky AS (2014). Ventilator-induced lung injury. Similarity and differences between children and adults. Am. J. Respir. Crit. Care Med. 190: 258-265.

Moraes L, Santos CL, Santos RS, Cruz FF, et al. (2014). Effects of sigh during pressure control and pressure support ventilation in pulmonary and extrapulmonary mild acute lung injury. Crit. Care 18: 474.

Muller-Redetzky HC, Will D, Hellwig K, Kummer W, et al. (2014). Mechanical ventilation drives pneumococcal pneumonia into lung injury and sepsis in mice: protection by adrenomedullin. Crit. Care 18: R73.

Park M, Pires-Neto RC and Nassar Jr AP (2014). Awaking, exercising, sitting, walking and extubating: moving on the paradigms for mechanically ventilated patients. Rev. Bras. Ter. Intensiva 26: 203-204.

Retamal J, Bergamini B, Carvalho AR, Bozza FA, et al. (2014). Non-lobar atelectasis generates inflammation and structural alveolar injury in the surrounding healthy tissue during mechanical ventilation. Crit. Care 18: 505 .

Roldan N, Goormaghtigh E, Perez-Gil J and Garcia-Alvarez B (2015). Palmitoylation as a key factor to modulate SP-Clipid interactions in lung surfactant membrane multilayers. Biochim. Biophys. Acta 1848: 184-191.

Saddy F, Sutherasan Y, Rocco PR and Pelosi P (2014). Ventilator-associated lung injury during assisted mechanical ventilation. Semin. Respir. Crit. Care Med. 35: 409-417.

Santos CD, Blanc C, Elahouel R, Prescott M, et al. (2014). Proliferation and migration activities of fibroblast growth 
Factor-2 in endothelial cells are modulated by its direct interaction with heparin affin regulatory peptide. Biochimie 107 (Pt. B): 350-357.

Shu YS, Tao W, Miao QB, Zhu YB, et al. (2014). Improvement of ventilation-induced lung injury in a rodent model by inhibition of inhibitory kappaB kinase. J. Trauma Acute Care Surg. 76: 1417-1424.

Simonato M, Baritussio A, Pioselli B, Ori C, et al. (2014). Surfactant protein C metabolism in human infants and adult patients by stable isotope tracer and mass spectrometry. Anal. Bioanal. Chem. 406: 6225-6233.

Tsushima K and Tatsumi K (2014). Noninvasive mechanical ventilation and neutrophil elastase inhibitor: A new potential approaching to acute hypoxemic failure. J. Crit. Care 29: 1124-1125.

Yang S, Stepien D, Hanseman D, Robinson B, et al. (2014). Substance P mediates reduced pneumonia rates after traumatic brain injury. Crit. Care Med. 42: 2092-2100. 